\title{
Channelized Hotelling Observers for Signal Detection in Stack-Mode Reading of Volumetric Images on Medical Displays with Slow Response Time
}

\author{
Ljiljana Platiša*, Student Member, IEEE, Cédric Marchessoux, Tom Kimpe, Ewout Vansteenkiste, Member, IEEE, \\ Aldo Badano, and Wilfried Philips, Senior Member, IEEE
}

\begin{abstract}
Volumetric medical images are commonly read in stack-browsing mode. However, previous studies suggest that slow temporal response of medical liquid crystal displays may degrade the diagnostic accuracy (lesion detectability) at browsing rates as low as $\mathbf{1 0}$ frames per second (fps). Recently, a multi-slice channelized Hotelling observer (msCHO) model was proposed to estimate the detection performance in $3 \mathrm{D}$ images. This implementation of the $\mathrm{msCHO}$ restricted the analysis to the luminance of a display pixel at the end of the frame time (end-of-frame luminance) while ignoring the luminance transition within the frame time (intra-frame luminance). Such an approach fails to differentiate between, for example, the commonly found case of two displays with different temporal profiles of luminance as long as their end-of-frame luminance levels are the same. In order to overcome this limitation of the msCHO, we propose a new upsampled msCHO (umsCHO) which acts on images obtained using both the intra-frame and the end-of-frame luminance information. The two models are compared on a set of synthesized 3D images for a range of browsing rates $(16.67,25$ and 50 fps). Our results demonstrate that, depending on the details of the luminance transition profiles, neglecting the intra-frame luminance information may lead to over- or underestimation of lesion detectability. Therefore, we argue that using the umsCHO rather than msCHO model is more appropriate for estimating the detection performance in the stack-browsing mode.
\end{abstract}

\section{INTRODUCTION}

V OLUMETRIC medical images are commonly read in stack browsing mode. Examples of 3D image modalities include MRI brain scans, CT scans of liver, 3D SPECT of bone, breast tomosynthesis, and many others. However, medical liquid crystal display (LCD) monitors today have a slow temporal response which could affect the diagnostic accuracy at higher browsing speeds. Though more investigation is required, previous studies of this effect suggest that slow

Manuscript received November 22, 2011.

*L. Platiša is with the group for Image Processing and Interpretation, Department of Telecommunications and Information Processing (TELIN-IPIIBBT), Ghent University, St-Pietersnieuwstraat 41, B-9000 Ghent, Belgium (telephone: +32 9264 3416, e-mail: Ljiljana.Platisa@telin.ugent.be).

A. Badano is with the Division of Imaging and Applied Mathematics, Office of Science and Engineering Labs, CDRH, FDA, Silver Spring MD 20993.

C. Marchessoux and T. Kimpe are with the Technology \& Innovation Group Healthcare Division, Barco N.V., President Kennedypark 35, 8500 Kortrijk, Belgium.

E. Vansteenkiste and W. Philips are with the group for Image Processing and Interpretation, the Department of Telecommunications and Information Processing (TELIN-IPI-IBBT), Ghent University, St-Pietersnieuwstraat 41, B9000 Ghent, Belgium. temporal response may degrade lesion (signal) detectability at browsing rates higher than 10 frames per second (fps) [1]-[5].

Recently, Platiša et al. [6] proposed a multi-slice channelized Hotelling observer (msCHO) model to estimate detection performance in 3D images inspected in stack browsing mode. In Ref. [3], this msCHO was used to estimate the effects of LCD's slow temporal response. In that work, the msCHO was restricted by design to the analysis of the luminance (gray level) of a display pixel at the end of the frame time (endof-frame luminance) while ignoring the information about the luminance transition during the frame time (intra-frame luminance). A similar approach was taken in the earlier study by Liang et al. [1] who used a conventional single-slice (2D) $\mathrm{CHO}$ applied to the image slice in which the signal was located. The analysis in Liang et al. also considered only the end-of-frame luminance values while ignoring the intra-frame luminance.

One weakness of the methods which ignore the intraframe luminance is their inability to differentiate between, for example, two displays with different profiles of luminance over time as long as their end-of-frame luminance levels are the same. Moreover, such methods are inadequate to capture the full effects of the techniques for response time compensation (overdrive technologies) used in today's high-end medical LCDs [4], [7], [8]. At the same time, studies with humans indicate a clear benefit of applying such techniques [5].

In this work, we extend the current implementation of the msCHO model to incorporate intra-frame luminance information. We refer to the new model as the upsampled msCHO, umsCHO. The two approaches, msCHO and umsCHO, are compared on a set of synthesized 3D images under browsing rates of 16.67, 25 and $50 \mathrm{fps}$. In order to investigate the influence of the luminance change profiles on the performance of the two models, we consider two different temporal response models of an LCD: a linear model, previously used in the studies by Liang et al. [1] and Platiša et al. [3], and the model proposed by Wang et al. [9], used in a more recent experimental study by Platiša et al. [4]. In addition, as a point of reference in evaluating the performance of the two models, we consider the case in which the browsing effects are ignored ("static" display mode).

The objectives of the present study are twofold: (1) to assess the significance of incorporating the intra-frame information 
when estimating detection performance in a stack-browsing reading scenario, and (2) to examine the role of the luminance transition form (profile) of the LCD on estimates of performance.

Our results demonstrate the benefit of integrating intraframe luminance information in the umsCHO. For our experimental conditions, the difference between msCHO and umsCHO input data caused by, respectively, excluding or including the intra-frame luminance information, is greatest for the browsing rate of $25 \mathrm{fps}$. At that rate, the msCHO outperforms the umsCHO and overestimates the detection performance at $25 \mathrm{fps}$. In addition, both msCHO and umsCHO capture a change in performance when the temporal profile of luminance is changed.

Given the conditions observed in human trials that suggest the luminance is a continuous function of time, the umsCHO could be a preferred human-like model design over the msCHO as it samples the time domain more frequently. While further investigation is needed to validate the agreement between the performance of the proposed umsCHO model and that of a human, our results indicate promise for the methodology to be used with clinically relevant image data as a metric of detection-based image quality.

The paper is organized as follows. In Section II, we first describe the synthetic image data from the study which represents the content to be displayed on a medical LCD. Next, we describe the two models of luminance change profiles which are used to simulate the effects of a medical LCD, as seen on the display. In Section III, we outline the basics of the existing msCHO model and explain the details of the umsCHO model proposed in this work. Our experimental results are presented and discussed in Section IV. Finally, concluding remarks are given in Section V.

\section{IMAGE AND DISPLAY SIMULATIONS}

\section{A. Synthetic multislice images}

Fig. 1 illustrates the simulated image data from this study. We generate a total of 2200 images, each containing 64 slices of $256 \times 256$ pixels in size. The background images are 3D clustered-lumpy backgrounds (CLB) [1] which in 2D have been shown to mimic the appearance of mammographic anatomical structure [10]. Half of the backgrounds are used as signal-absent images while the remaining half of 1100 backgrounds are used to create signal-present images. The signal is a simulated 2D designer nodule [1], [11] inserted in the central image slice (slice 32). In our model observer experiments, 1000 pairs of signal-present and signal-absent images are used as training data and 100 image pairs are used as test data. The details are given in Section III-C.

The images described here are referred to as "static" or preLCD images as they do not take into account the effects of the display.

\section{B. Temporal response models for medical LCDs}

When a pre-LCD image is shown on a display we refer to it as a post-LCD image. In our study, we restrict the analysis of the display's effects to the response time of liquid
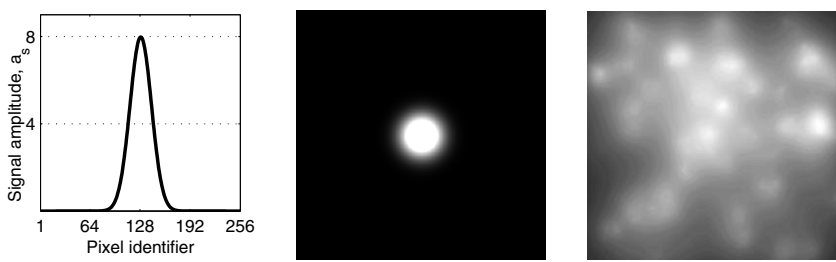

Fig. 1. Simulated image data. (Left) Contrast profile of the $2 \mathrm{D}$ signal with amplitude $a_{s}=8$. (Middle) 2D signal image. (Right) An example signalabsent image slice used in the study. The parameters of 3D CLB background are: mean number of clusters $K=160$, mean number of blobs per cluster $N=20$, and and characteristic lengths $L_{x}=3, L_{y}=2, L_{z}=3$. The image size is $256 \times 256 \times 64$ pixels. For signal-present images, the signal is located in slice 32 out of 64 .
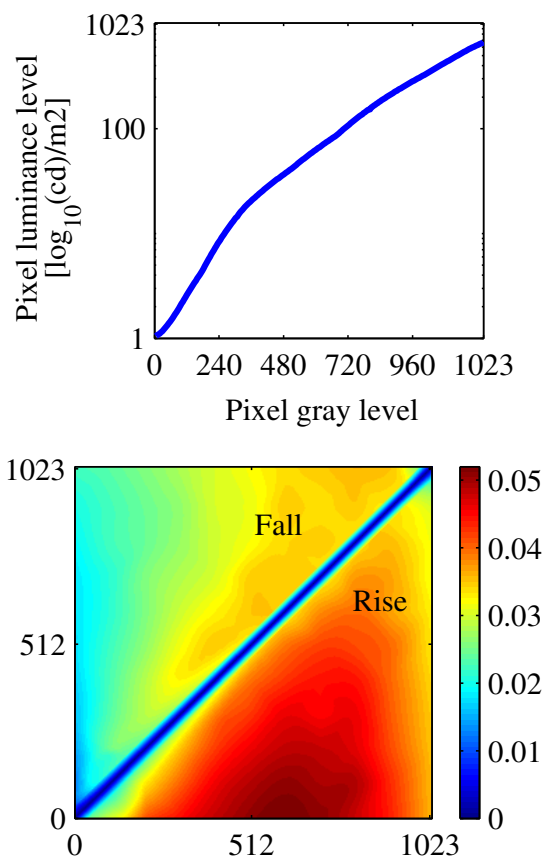

Fig. 2. Temporal response model of the five-million-pixel medical LCD used in the study. (Top) Luminance response curve of the display. (Bottom) Matrix of the response time of the display.

crystal cells, the temporal response of the LCD. In all other aspects including spatial noise and contrast variability due to viewing angle, the display performance is considered ideal. Thus, the difference between our pre- and post-LCD image is in the luminance (mapping of gray levels) of their pixels (refer to Fig. 3 for an illustration). The display parameters in our display simulations correspond to a 5MP 10-bit medical grayscale LCD for full-field digital mammography [4]. The luminance response curve and matrix of the liquid crystal director reorientation times for the given display are shown in Fig. 2.

If we are browsing through the image at a rate of $f_{\text {browse }}$, each frame is displayed during the frame time $T_{\text {frame }}=T_{\text {browse }}=1 / f_{\text {browse }}$. In our experiments $f_{\text {browse }} \in$ $\{16.67,25,50\}$ fps, while the LCD refresh rate is $f_{\text {refresh }}=$ $50 \mathrm{~Hz}\left(T=T_{\text {refresh }}=20 \mathrm{~ms}\right)$. Note that the highest feasible browsing rate is limited to the refresh rate of the display ( $\left.f_{\text {browse }} \leq f_{\text {refresh }}\right)$, that is, we can only view as many slices per second as the number of times the display screen is 


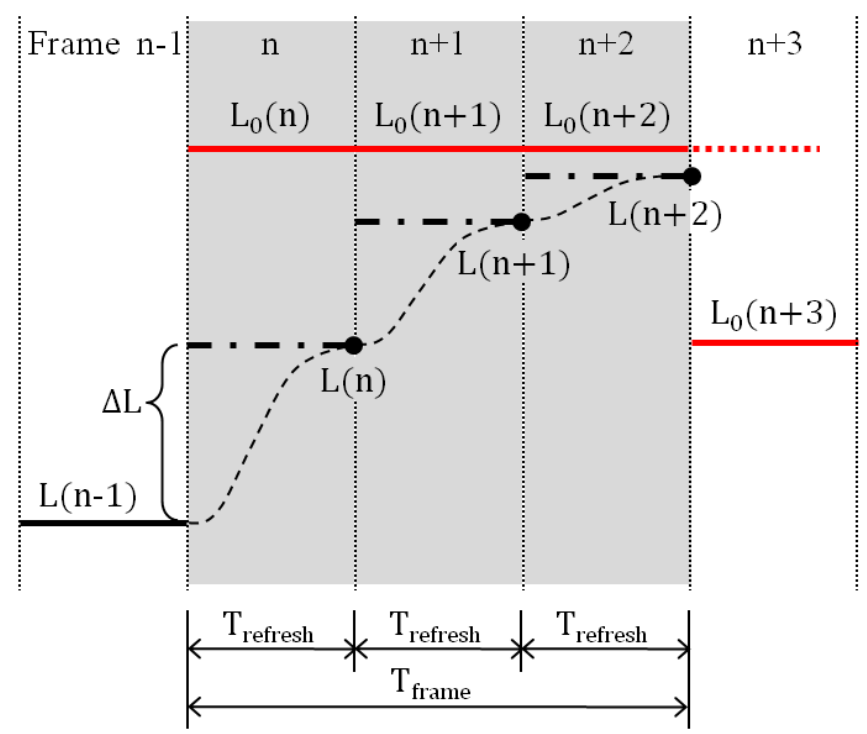

Fig. 3. Pixel luminance change at different post-LCD frames. $L(n-1)$ is the achieved luminance of pixel $(i, j)$ at frame $n-1$. At frame $n$, the target luminance level is $L_{0}(n)$. Because of slow temporal response, the achieved luminance at frame $n$ is $L(n) \leq L_{0}(n)$. Similar applies to the following frames $n+1, n+2$ and $n+3$.

refreshed per second.

We refer to Fig. 3 to explain the effects of the slow temporal response of an LCD. The figure illustrates transition from the luminance level $L(n-1)$ achieved at the end of frame $n-1$ to the target luminance level $L_{0}(n)$ in the following frame $n$. Depending on the extent of the difference between the achieved $L(n-1)$ and the target $L_{0}(n)$ luminance level, and depending on the LCD response time for a given transition (from $L(n-1)$ to $L_{0}(n)$ ), it may take multiple display refresh intervals $T_{\text {refresh }}$ for a given LCD cell to achieve the target luminance level $L_{0}(n)$ and thus complete the target transition. Moreover, in cases where the frame time $T_{\text {browse }}$ is smaller than the corresponding response time of the LCD cell, the target luminance level $L_{0}(n)$ will not even be achieved, as in the example from Fig. 3 where $L(n+2)<L_{0}(n+2)$. These "delayed" or "incomplete" changes in target luminance transitions result in the reduced effective luminance contrast of details in medical images. Consequently, the detection performance in stack-mode reading of volumetric images may be degraded, as previously shown in the literature [1], [3], [4].

For the purpose of further analysis, we introduce the terms of intra- and end-of-frame luminance that are achieved luminances. For simplicity, let us consider the case where browsing speed is $f_{\text {browse }}=25 \mathrm{fps}\left(T_{\text {browse }}=2 T=40\right.$ ms). Here, the levels of luminance $L$ achieved at $1 T, 3 T, \ldots$ are referred to as the intra-frame luminances $L_{i n}$ while $L$ at $2 T, 4 T, \ldots$ are the end-of-frame luminances $L_{e o f}$ (see also Fig. 4). Importantly, because of the slow temporal response of the LCD, $L_{\text {in }} \leq L_{\text {out }}$ when the achieved luminance is lower than the target luminance, or $L_{\text {in }} \geq L_{\text {out }}$ when the achieved luminance is higher than the target luminance.

In this study we explore two different models for temporal response of the LCD (see Fig. 4): $l_{p 1}$, a physics-based profile model proposed by Wang et al. [9], and $l_{p 2}$, a linear profile

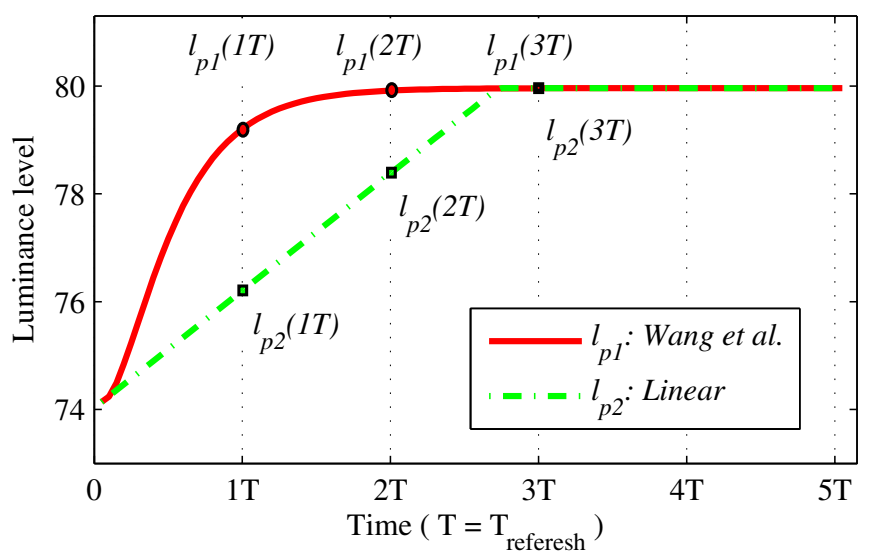

Fig. 4. Pixel luminance change at different post-LCD frames. Two luminance transition models are depicted: $l_{p 1}$ corresponding to the work of Wang et al. [9] and $l_{p 2}$ assuming linear dependence of change in luminance level with time.

model used in Refs. [1], [3]. We note from Fig. 4 that the level of luminance achieved at the end of each display refresh interval largely depends on the form of the luminance transition curve: $\left(1-\exp \left(-2 t / \tau_{0}\right)\right.$ in the case of $l_{p 1}$, or $t / \tau_{0}$ for $l_{p 2}$, where $\tau_{0}$ denotes the reorientation time of the liquid crystal director. Here, the values of parameter $\tau_{0}$ are determined using measured values from the matrix of the response time shown in the bottom of Fig. 2 .

We review here the steps involved in the process of image data simulations in our study. We start by generating the preLCD images in grayscale space, as explained in Section II-A. Next, the pre-LCD image pixel values are converted to luminance values (target luminance images) using the luminance response curve from Fig. 2. Then, we apply the two temporal response models of a medical LCD described in this section to obtain two post-LCD images in luminance space (achieved luminance images). Finally, the post-LCD images are converted back to the grayscale space, again using the luminance response curve from Fig. 2, and the model observers are applied to estimate the detection performances for both image sets.

\section{MODEL OBSERVERS FOR MULTI-SLICE IMAGES}

\section{A. Multi-slice $\mathrm{CHO}$ (msCHO)}

Recent results on using model observers to assess effects of slow temporal response of medical LCDs on the detection performance reported Platisa et al. [3], [4] suggest that the msCHO model, especially the $\mathrm{msCHO}_{b}$ variant from Ref. [6], could be used for human-like assessment of multi-slice images. We outline here the basic principles of the $\mathrm{msCHO}_{b}$ model design (hereafter referred as msCHO). The details can be found in Ref. [6].

The msCHO performs the detection task in a two stage process, as illustrated in Fig. 5. In the first stage, the observer pre-processes the image stack in planar view ( $x y$-plane), slice after slice, and buffers the scores obtained for each slice. To model this, first, a filter bank of 2D channels, $\mathbf{U}=\left[\mathbf{U}_{(1)}, \ldots, \mathbf{U}_{(P)}\right]$, applied on the grayscale image pixel 


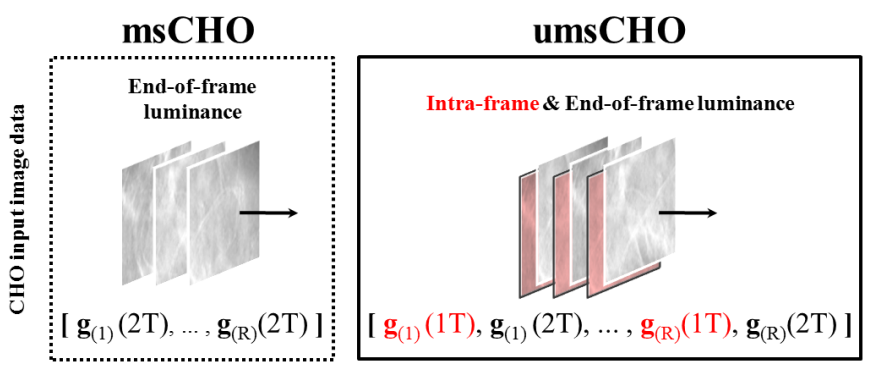

Fig. 6. Upsampled msCHO (umsCHO) model design adapted from an existing msCHO model [6]. While the $\mathrm{msCHO}$ is limited to the end-offrame post-LCD image data, the umsCHO model has access to both the intraframe and the end-of-frame post-LCD image data. Specifically, the illustration assumes a browsing rate of $f_{\text {browse }}=25 \mathrm{fps}$ under the display refresh rate of $f_{\text {refresh }}=50 \mathrm{~Hz}$; hence each image frame (slice) is displayed over the frame time $T_{\text {frame }}=2 T_{\text {refresh }}=2 T$. There, the msCHO acts only on image pixel values at frame time intervals: $T_{\text {frame } 1}=2 T, T_{\text {frame } 2}=4 T$, $\ldots$, while umsCHO is aware of image values at each refresh time interval $T=T_{\text {refresh }}$.

values of each slice, $\left[\mathbf{g}_{(1)}, \ldots, \mathbf{g}_{(R)}\right]$, to get the channelized slice data, $\mathbf{v}_{\text {planar }}=\left[\mathbf{v}_{(1)}, \ldots, \mathbf{v}_{(R)}\right]$. Here, $P$ denotes the number of channels and $R$ denotes the number of adjacent slices in the region of interest (ROI) in which the signal is located. The signal in our images only exists in the central image slice and thus the size of ROI is varied among 3, 5 and 7 slices around the signal slice. The channels used in the study are the first $P=10$ dense difference-of-Gaussian (DDOG) channels. The values of our DDOG channel parameters correspond to those used in the study of Abbey and Barrett [12] which showed to closely track human observer performance. Next, the channelized slice data, $\mathbf{v}_{\text {planar }}$, are used to build a test statistic for each of the $R$ slices. This step corresponds to the conventional $2 \mathrm{D}-\mathrm{CHO}$ characterized by its template $\mathbf{w}_{C H O}$ and applied on each $R$ slices, giving $t_{\text {planar }}=\left[t_{(1)}, \ldots, t_{(R)}\right]$. Here, the $\mathbf{w}_{C H O}$ template is estimated using the training image data of the slices that contain the signal, $\mathbf{g}^{\mathrm{TR}}(R / 2-1)$.

In the second stage, the observer integrates the information in the $z$-direction to result in the final stack test statistic (image rating) $t_{m s C H O}$. In terms of the model, $t_{\text {planar }}$ is used as input to the Hotelling observer [13] (HO) with the template $\mathbf{w}_{H O}$ which then produces the $t_{m s C H O}$.

\section{B. Novel observer model: upsampled msCHO (umsCHO)}

Existing literature reports on studying effects of slow LCDs on signal detectability [1], including the msCHO strategy [3], limit their analysis to the end-of-frame $L_{\text {eof }}$ luminance values, that is, to their corresponding grayscale values $g_{e o f}$. In this work, we incorporate additional data given by the intra-frame information, $L_{i n}$ or $g_{i n}$. For this purpose, the msCHO model design is modified to process image values after each $T_{\text {referesh }}$ interval, as illustrated in Fig. 6. The new model is named upsampled $\mathrm{msCHO}$ (umsCHO).

Compared to the msCHO, the new umsCHO model has access to image information sampled over more finely spaced intervals of time and thus we expect it to make more accurate estimates of the detectability in post-LCD images. Moreover, the conditions observed in human trials, where the luminance is not a discrete but a continuous function of time, are better approximated by the umsCHO sampling the time domain more frequently than the msCHO. The two models are explored in more detail in our experiments described next.

\section{Design of model observer experiments}

For each model observer design, msCHO and umsCHO, we conducted the experiments for seven different image setups: pre-LCD images, and post-LCD images at three different browsing rates $(16.67,25$ and $50 \mathrm{fps}$ ) each simulated with two different temporal response models of an LCD.

All experimental designs are multi-reader multi-case (MRMC) studies [14] with 5 readers per browsing rate, each trained on an independent subset of 200 training image pairs and applied on a unique set of 100 test image pairs. The training and the testing images do not overlap. In comparing the observer performances, we use the detection signal-to-noise ratio (SNR) computed from the observer's test statistics [13]. The corresponding error bars are estimated using MRMC-type of bootstrap analysis [14], [15] where each bootstrap iteration selects a set of readers and cases.

\section{RESULTS AND DISCUSSION}

In Fig. 7, we show the results of our display simulations for the two temporal response models from Fig. 4. The changes in intensity of the central pixel in the $x y$-plane are shown for an example signal-present image sequence. The three plots correspond to three different browsing rates, $f_{\text {browse }}$ : 16.67, 25 , and 50 fps. Next to the changes in intensity of the postLCD images, each plot depicts the intensity profile of the static (pre-LCD) image, to serve as a reference for comparative analysis. Two main observations can be made from these plots: (1) the profile of the luminance transitions over time has an impact on the intra-frame pixel values achieved while browsing through an image stack, and (2) as the browsing rate increases (from 16.67 to $50 \mathrm{fps}$ ), the error in image values introduced by ignoring the intra-frame image values increases.

In Fig. 8 we show the results of the msCHO and umsCHO experiments when the size of ROI is 7 . The top plot depicts the results for $l_{p 1}$ model of luminance transitions proposed by Wang et al. [9], and the bottom plot gives the results for $l_{p 2}$ linear luminance transition model. Indicated error bars correspond to \pm 2 standard deviations estimated using bootstrap with 1000 re-samplings. Overall, the SNR trends suggest degradation in the detection performance of the observers as browsing speed increases, which is consistent with the results of earlier similar studies [1], [3], [4].

However, given the luminance change profiles from Fig. 4 and the changes in intensity of the central signal pixel illustrated in Fig. 7, we expect the detectability to decrease as the browsing rate increases. This expectation is satisfied by umsCHO but not by msCHO. For our experimental data (see Fig. 7), the difference between msCHO and umsCHO input data is greatest for browsing rate of 25 fps: msCHO only knows the nearly asymptotic luminance values achieved at $2 \mathrm{~T}$, while umsCHO is also aware of the much lower values achieved by $1 \mathrm{~T}$. This causes the msCHO to overestimate the detection performance at 25 fps. 


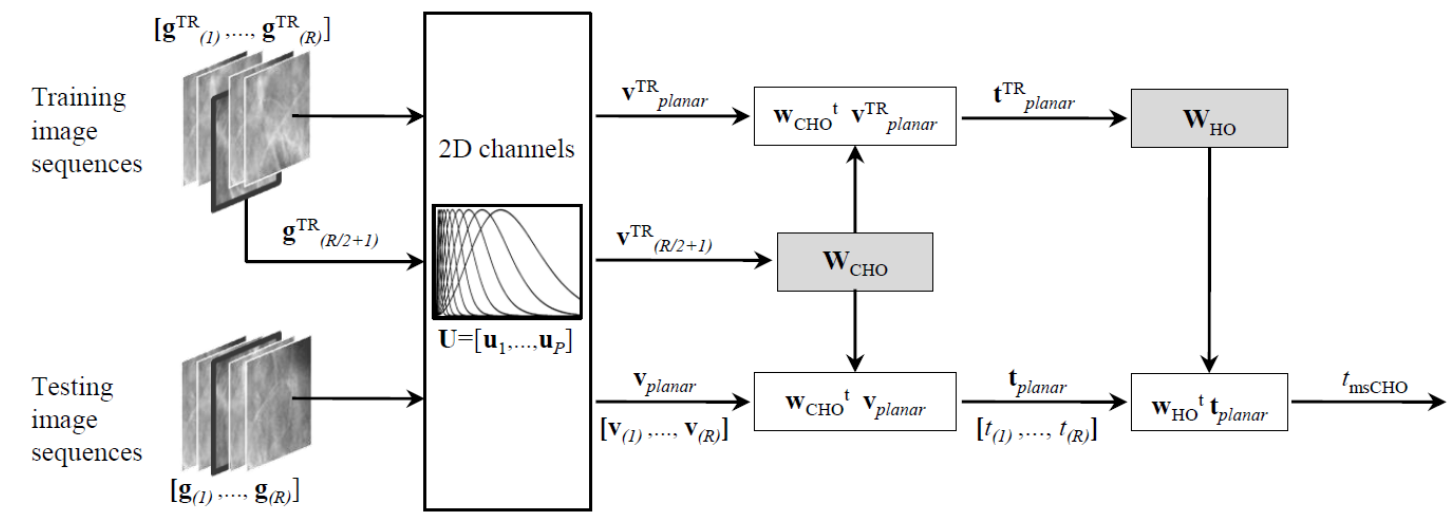

Fig. 5. Multi-slice channelized Hotelling observer (msCHO) model [6].
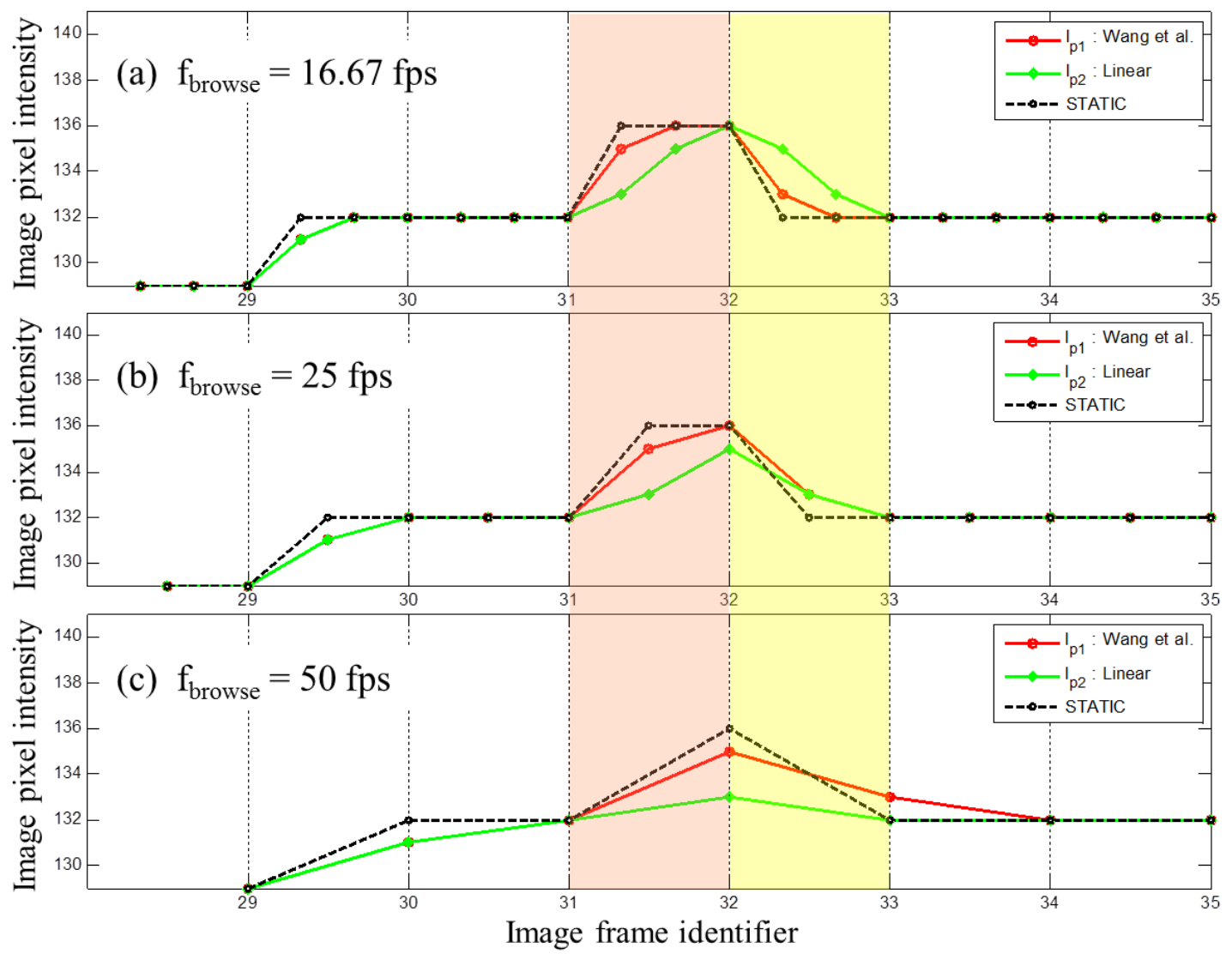

Fig. 7. The intensity profile of the central pixel in the image slice is depicted across one image stack. The browsing speed is varied from (a) $f_{b r o w s e}=16.67$ fps, through (b) $f_{\text {browse }}=25$ fps and up to (c) $f_{\text {browse }}=50$ fps. For each browsing speed, two luminance transition models are considered, $l_{p 1}$ and $l_{p 2}$ (see Fig. 4). As a reference, the dashed line in each plot represents the intensity profile of the static (pre-LCD) image. Compared to their pre-LCD values, post-LCD images exhibit a tailing effect in the slices around the signal. This is caused by slow temporal response of the display.

Finally, by comparing the SNR trends for the two luminance profiles, $l_{p 1}$ and $l_{p 2}$, we notice that the detection performances estimated by either msCHO or umsCHO are lower for the linear $l_{p 2}$ profile, especially at higher browsing rates - at 25 and $50 \mathrm{fps}$, the difference between SNR values for $l_{p 1}$ and for $l_{p 2}$ is approximately 2 . Therefore, we find that an adequate choice of the luminance model in simulations of the effects of the LCD luminance temporal transitions on the detection performance is essential.

\section{CONCLusions}

Our results demonstrate the impact of incorporating the intra-frame information when estimating the detection performance for images inspected in the stack-browsing mode. Integrating the intra-frame information into a model observer allows the model to be aware of LCD temporal luminance variations. Depending on the details of the luminance profile, neglecting the intra-frame luminance information may lead to under- or overestimation of signal detectability. 

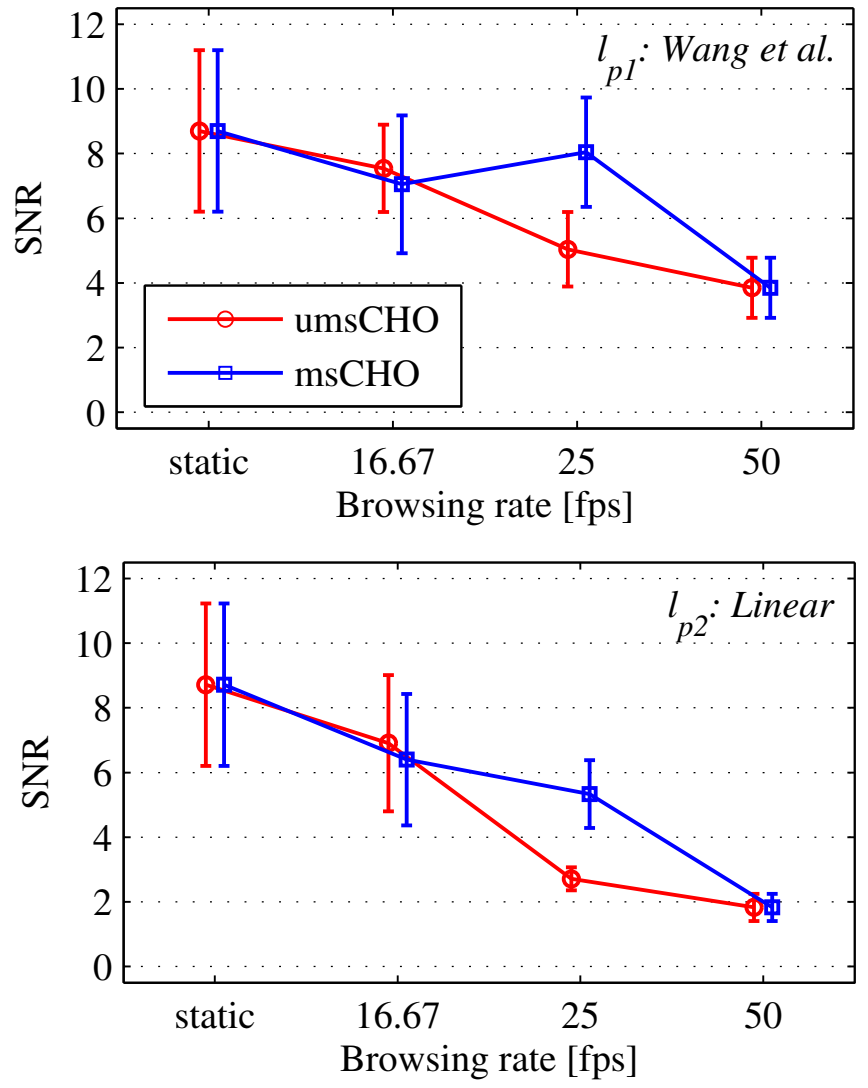

Fig. 8. Detection performance of the umsCHO compared to the msCHO for two different luminance transition profiles: $l_{p 1}$ (top) and $l_{p 2}$ (bottom); see also Fig. 4.

\section{REFERENCES}

[1] H. Liang, S. Park, B. Gallas, K. Myers, and A. Badano, "Image browsing in slow medical liquid crystal displays," Acad. Radiol., vol. 15, no. 3, pp. 370-82, Mar 2008.

[2] A. Badano, "Effect of slow display on detectability when browsing large image datasets," J. Soc. Inf. Display, vol. 17, no. 11, pp. 891-896, Nov 2009.

[3] L. Platiša, B. Goossens, E. Vansteenkiste, A. Badano, and W. Philips, "Using channelized hotelling observers to quantify temporal effects of medical liquid crystal displays on detection performance," in Proc. SPIE MI, vol. 7627, 2010, p. 76270U.

[4] L. Platiša, C. Marchessoux, B. Goossens, and W. Philips, "Performance evaluation of medical led displays using $3 \mathrm{~d}$ channelized hotelling observers," in Proc. SPIE MI, vol. 7966, 2011, p. 79660T.

[5] C. Marchessoux, N. Vivien, A. Kumcu, and T. Kimpe, "Validation of a new digital breast tomosynthesis medical display," in Proc. SPIE MI, vol. 7966, 2011, p. 79660R.

[6] L. Platiša, B. Goossens, E. Vansteenkiste, S. Park, B. D. Gallas, A. Badano, and W. Philips, "Channelized hotelling observers for the assessment of volumetric imaging data sets," J. Opt. Soc. Am. A, vol. 28, no. 6, pp. 1145-1163, Jun 2011.

[7] R. I. McCartney, "48.3: A liquid crystal display response time compensation feature integrated into an lcd panel timing controller," SID Symposium Digest of Technical Papers, vol. 34, no. 1, pp. 1350-1353, 2003.

[8] S. Kumar, M. Biswas, and T. Nguyen, "Analysis of the Response Time Compensation system for Liquid Crystal Displays," European Signal Processing Conference, vol. 13, 2005.

[9] H. Wang, T. Wu, X. Zhu, and S. Wu, "Correlations between liquid crystal director reorientation and optical responsetime of a homeotropic cell," J. Appl. Phys., vol. 95, no. 10, pp. 5502-5508, May 2004.

[10] F. O. Bochud, C. K. Abbey, and M. P. Eckstein, "Statistical texture synthesis of mammographic images with clustered lumpy backgrounds," Opt. Express, vol. 4, pp. 33-43, Jan 1999.
[11] A. E. Burgess, F. L. Jacobson, and P. F. Judy, "Human observer detection experiments with mammograms and power-law noise," Med. Phys., vol. 28, pp. 419-437, Apr 2001.

[12] C. K. Abbey and H. H. Barrett, "Human- and model-observer performance in ramp-spectrum noise: effects of regularization and object variability," J. Opt. Soc. Am. A, vol. 18, no. 3, pp. 473-488, Mar 2001

[13] H. H. Barrett and K. J. Myers, Foundations of Image Science. John Wiley and Sons, New York, 2004.

[14] B. D. Gallas, A. Bandos, F. W. Samuelson, and R. F. Wagner, "A Framework for Random - Effects ROC Analysis: Biases with the Bootstrap and Other Variance Estimators," Communications in Statistics - Theory and Methods, vol. 38, no. 15, pp. 2586-2603, Jul 2009.

[15] S. V. Beiden, R. F. Wagner, and G. Campbell, "Components-of-variance models and multiple-bootstrap experiments: an alternative method for random-effects, receiver operating characteristic analysis." Acad. Radiol., vol. 7, no. 5, pp. 341-349, May 2000 